\title{
Menopausa e síndrome metabólica
}

\author{
Menopause and metabolic syndrome
}

Ricardo M. R. Meirelles ${ }^{1}$

\section{RESUMO}

A incidência de doença cardiovascular aumenta consideravelmente após a menopausa. Um dos motivos para o crescente risco cardiovascular parece ser determinado pela síndrome metabólica, da qual todos os componentes (obesidade visceral, dislipidemia, hipertensão arterial e distúrbio do metabolismo glicídico) se associam à maior incidência de coronariopatia. Após a menopausa, a síndrome metabólica é mais prevalente do que na pré-menopausa, podendo ter importante papel na ocorrência de infarto do miocárdio e outras morbidades ateroscleróticas e cardiovasculares. A obesidade, componente primordial da síndrome metabólica, se associa ainda ao aumento da incidência de câncer de mama, endométrio, intestino, esôfago e rim. 0 tratamento da síndrome metabólica se baseia na mudança de hábitos de vida e, quando necessário, no emprego de medicação dirigida aos seus componentes. Na presença de sintomas de síndrome do climatério, a terapia hormonal, quando indicada, concorrerá também para a melhora da síndrome metabólica. Arq Bras Endocrinol Metab. 2014;58(2):91-6

\section{Descritores}

Menopausa; síndrome metabólica; terapia hormonal da menopausa; resistência insulínica; estrogênios

\section{ABSTRACT}

The incidence of cardiovascular disease increases considerably after the menopause. One reason for the increased cardiovascular risk seems to be determined by metabolic syndrome, in which all components (visceral obesity, dyslipidemia, hypertension, and glucose metabolism disorder) are associated with higher incidence of coronary artery disease. After menopause, metabolic syndrome is more prevalent than in premenopausal women, and may plays an important role in the occurrence of myocardial infarction and other atherosclerotic and cardiovascular morbidities. Obesity, an essential component of the metabolic syndrome, is also associated with increased incidence of breast, endometrial, bowel, esophagus, and kidney cancer. The treatment of metabolic syndrome is based on the change in lifestyle and, when necessary, the use of medication directed to its components. In the presence of symptoms of the climacteric syndrome, hormonal therapy, when indicated, will also contribute to the improvement of the metabolic syndrome. Arq Bras Endocrinol Metab. 2014;58(2):91-6

Keywords

Menopause; metabolic syndrome; hormone therapy in the menopause; insulin resistance; estrogens
${ }^{1}$ Instituto Estadual de Diabetes e Endocrinologia Luiz Capriglione (IEDE), Rio de Janeiro, RJ, Brasil

\section{INTRODUÇÃO}

\section{A} s doenças cardiovasculares são a maior causa de mortalidade em ambos os sexos e sua incidência em mulheres no menacme é menor do que nos homens de mesma idade, diminuindo progressivamente essa diferença com o envelhecimento e o advento da menopausa (1). Considerando-se apenas o sexo feminino, o estudo de Framingham mostrou que a incidência anual de doença cardiovascular em mulheres com menos de 55 anos na pré-menopausa, quando comparada à de mulheres, na mesma faixa etária, nas quais já ocorrera a menopausa, foi cerca de 50\% menor (2). A falência ovariana parece, portanto, ser um divisor de águas, em termos de risco cardiovascular.
Um dos fatores que aumentam o risco de mortalidade, em homens e mulheres, é a síndrome metabólica. A Third National Health and Nutrition Examination Survey (NHANES III) demonstrou que a síndrome metabólica se associa a maior risco de mortalidade para mulheres na pós-menopausa do que para homens ou mulheres na pré-menopausa (3). Diversos trabalhos mostram maior prevalência de síndrome metabólica em mulheres na pós-menopausa em relação àquelas na pré-menopausa (4-8). Isso pode ser consequência direta da falência ovariana ou resultar de alterações metabólicas promovidas pelo aumento de gordura visceral secundária à diminuição dos estrogênios, não estando claro se a menopausa aumenta o risco cardiovascular em todas as 
mulheres ou apenas nas portadoras de síndrome metabólica (9). Há quem sugira que se avaliem e tratem todos os fatores de risco cardiovascular, sem se preocupar se a paciente preenche ou não os critérios diagnósticos de síndrome metabólica (10).

A prevalência de síndrome metabólica em mulheres na pós-menopausa é de $22 \%$ a $69 \%$, variando de um país para outro e com a metodologia empregada (Tabela 1 ) (4-8). Uma pesquisa sueca, entretanto, não encontrou diferença na prevalência de síndrome metabólica antes e após a menopausa, observando, entretanto, uma associação com o nível de educação, sendo mais prevalente nas mulheres com menor escolaridade (11). O nível educacional mais elevado permite que se identifiquem os riscos associados a obesidade, hipertensão e alterações do metabolismo glicídico e lipídico, bem como o conhecimento necessário para adotar as medidas preventivas apropriadas.

É objetivo da presente revisão analisar a inter-relação entre menopausa e os componentes da síndrome metabólica, suas consequências e as intervenções capazes de evitá-las.

\section{RESISTÊNCIA INSULÍNICA, INTOLERÂNCIA À GLICOSE E DIABETES}

A síndrome metabólica representa a associação de diversos fatores de risco cardiovascular, como hipertensão arterial, distúrbios do metabolismo glicídico e lipídico, além de obesidade visceral. Foram propostos três crité- rios para o diagnóstico da síndrome metabólica: o da Organização Mundial de Saúde (OMS), o da National Cholesterol Education Program - The Adult Treatment Panel III (NCEP- ATP III) e o da International Diabetes Federation (IDF) (12). Todos se baseiam nos fatores de risco cardiovascular citados, exceto o primeiro deles, da OMS, publicado em 1998, que também inclui a presença de albuminúria maior que $20 \mathrm{mcg} /$ minuto ou uma relação albumina/creatinina urinária maior que $30 \mathrm{mg} / \mathrm{g}$ (13).

A resistência insulínica é considerada a base fisiopatológica da síndrome metabólica (14) desde a sua primeira descrição por Reaven, que a chamou de Síndrome X (15). A obesidade visceral é um sinal clínico da resistência insulínica, embora esta possa existir desacompanhada de aumento de peso. Os três critérios utilizados para diagnóstico da síndrome metabólica, entretanto, incluem o aumento do índice de massa corporal (IMC) ou da circunferência de cintura. Além de contribuir para a intolerância à glicose e diabetes, hipertensão arterial, aumento dos triglicerídeos e diminuição do colesterol HDL, a resistência insulínica se associa a aumento do ácido úrico no sangue, diminuição do volume das partículas de colesterol LDL, aumento da lipemia pós-prandial, retenção de sódio, aumento da atividade do sistema nervoso simpático, aumento do fibrinogênio, do inibidor do ativador do plasminogênio tipo l (PAI-1), disfunção endotelial e, em mulheres no menacme, à síndrome dos ovários policísticos (16).

Tanto em homens como em mulheres a prevalência de diabetes aumenta a partir dos 50 anos, sendo

Tabela 1. Prevalência de síndrome metabólica em mulheres na pré e pós-menopausa

\begin{tabular}{|c|c|c|c|c|c|}
\hline \multirow{2}{*}{ Autor, ano } & \multirow{2}{*}{ País } & \multirow{2}{*}{$\begin{array}{c}\text { Critério para } \\
\text { diagnóstico da } \\
\text { sindrome metabólica }\end{array}$} & \multirow{2}{*}{$\mathbf{N}$} & \multicolumn{2}{|c|}{ Prevalência \% } \\
\hline & & & & Pré-menopausa & Pós-menopausa \\
\hline Mesch, 2006 & Argentina & NCEP-ATP III & 60 & 0,0 & 22,0 \\
\hline Marjani, 2012 & Irã & NCEP-ATP III & 100 & N.D. & 31,0 \\
\hline Piché, 2006 & Canadá & NCEP-ATP III & 108 & N.D. & 31,0 \\
\hline Ponholzer, 2008 & Áustria & IDF & 538 & 8,5 & 32,6 \\
\hline Ding, 2007 & China & IDF & 225 & N.D. & 37.3 \\
\hline Hidalgo, 2006 & Equador & NCEP-ATP III & 325 & N.D. & 41,5 \\
\hline Deibert, 2007 & Alemanha & NCEP-ATP III & 72 & 22,7 & 42,0 \\
\hline Figueiredo Neto, 2010 & Brasil & IDF & 323 & N.D. & 49,8 \\
\hline Eshtiaghi, 2010 & Irã & NCEP-ATP III & 940 & 18,3 & 53,5 \\
\hline Kim, 2007 & Coreia & NCEP-ATP III & 2671 & 13,8 & 54,6 \\
\hline Pandey, 2010 & Índia & IDF & 498 & 45,0 & 55,0 \\
\hline Heidari, 2010 & Irã & NCEP-ATP III & 1290 & 44,9 & 64,3 \\
\hline Ainy, 2007 & Irã & NCEP-ATP III & 1871 & 53,0 & 69,0 \\
\hline
\end{tabular}

N: número de participantes; IDF: International Diabetes Federation; NCEP-ATP III: Cholesterol Education Program - The Adult Treatment Panel III; N.D. = não disponível. 
a elevação mais acentuada nas mulheres, em que essa prevalência atinge quase o dobro da masculina entre 60 e 69 anos (17). Possivelmente a menopausa contribua para essa prevalência aumentada.

A terapia hormonal da menopausa por via oral pode ser preferível nas mulheres com síndrome metabólica ou diabetes tipo 2, por contribuir para diminuição da resistência insulínica (18).

\section{OBESIDADE}

As definições de síndrome metabólica da NCEP-ATP III (19) e da IDF (20) estabelecem a circunferência da cintura, em homens e mulheres, como índice de obesidade central, sendo que a IDF fixa valores de corte segundo a etnia ou país de origem (europeus, sul-asiáticos, chineses, japoneses, centro e sul-americanos, africanos subSaara e árabes). Para a OMS (13) podem ser considerados índices de obesidade o IMC maior que $30 \mathrm{~kg} / \mathrm{m}^{2}$ ou a relação cintura-quadril (maior que 0,90 para homens ou 0,85 para mulheres).

O tecido adiposo, além de servir como reserva de energia, produz uma série de citocinas inflamatórias, como o fator de necrose tumoral alfa (TNF- $\alpha$ ), a interleucina-6 (IL-6) e a proteína quimiotática de monócitos l (MCP-1) (21). O excesso de peso após a menopausa acentua a resistência insulínica e contribui também para o aumento da leptina e da resistina, bem como redução da adiponectina e da ghrelina, o que aumenta ainda mais o risco cardiovascular (22). Outra adipocitocina que se revelou elevada em mulheres pós-menopausa com síndrome metabólica foi a visfatina, observando-se uma elevação tanto maior quanto maior o número de componentes da síndrome metabólica identificados, tendo sido especulado um eventual papel dessa adipocitocina na fisiopatologia da síndrome (23).

Embora as mulheres tenham maior proporção de gordura corporal do que os homens, nelas predomina o depósito subcutâneo, com maior acúmulo em quadril e coxas. A gordura acumulada em quadril e coxas, diferentemente da visceral, se associa a um perfil glicídico e lipídico mais favorável, bem como a uma diminuição do risco cardiovascular e metabólico, com níveis mais elevados de adiponectina e redução das citocinas inflamatórias (24). Antes da menopausa a atividade da lipase lipoproteica é maior na região femoral do que na abdominal ou mamária e essa predominância deixa de existir após a menopausa, propiciando uma modificação da distribuição de gordura corporal, com acúmulo no tronco (25). Os estrogênios inibem o acúmulo de gordura abdominal antes da menopausa (26) e sua diminuição facilita o aumento da gordura central.

A medida da quantidade e distribuição da gordura corporal por raios-X de dupla energia (DXA) mostra que as mulheres na pós-menopausa têm $20 \%$ mais gordura corporal do que as na pré-menopausa e que o percentual de gordura abdominal é de, respectivamente, $42,1 \%$ e $38,3 \%$ (27). Embora a distribuição central de gordura seja evidente na mulher após a menopausa, não há consenso sobre se a falência ovariana se associa a aumento de peso (28). O uso de terapia hormonal estrogênica é capaz de evitar o acúmulo de gordura visceral, independente do efeito sobre os lipídios e lipoproteínas circulantes e sem que haja perda significativa de peso (29). A redução do metabolismo basal que ocorre após a menopausa, juntamente com a diminuição da atividade física e do gasto energético, a redução da massa muscular e o acúmulo de gordura abdominal, provavelmente contribui para o risco aumentado de doença cardiovascular (30).

Além do aumento de risco cardiovascular, a obesidade se associa a maior risco de malignidades, em especial o câncer de mama, endométrio, intestino, esôfago e rim (31). Um estudo longitudinal mostrou a relação da síndrome metabólica, especialmente de alguns de seus componentes, como hiperglicemia, hipertrigliceridemia e hipertensão arterial diastólica, com o risco de câncer de mama (32), sendo que a razão de chance desse risco para mulheres com três ou mais componentes da síndrome metabólica é de 1,75 (IC a 95\%: 1,37-2,22) (33). A única vantagem que a mulher obesa na pós-menopausa parece ter é a menor incidência de osteoporose (34).

Os componentes mais prevalentes da síndrome metabólica em 118 mulheres iranianas pós-menopausa foram a obesidade e a hipertensão arterial, sendo que $15 \%, 13,3 \%$ e $1,8 \%$ delas apresentavam, respectivamente, três, quatro e cinco critérios para síndrome metabólica (35). Quanto maior o número de componentes presentes, maior o risco cardiovascular.

O tratamento da obesidade deve se basear na orientação alimentar adequada e na realização de atividade física regular, observando-se que a perda de $5 \%$ a $10 \%$ do peso corporal já se acompanha de melhora metabólica e cardiovascular (36) e a musculação é capaz de melhorar a resistência insulínica em mulheres na pós-menopausa (37). A terapia hormonal da menopausa não piora o controle metabólico de mulheres que já sejam portadoras de intolerância à glicose ou diabetes tipo 2 (38). 


\section{DISLIPIDEMIA}

A falência ovariana se associa a aumento dos triglicerídeos, do LDL colesterol e suas frações pequenas e densas, diminuição do HDL colesterol e aumento da Lp(a), gerando um perfil aterogênico, compatível com a síndrome metabólica (39). Com relação ao HDL colesterol, é mais evidente a redução da subfração HDL2, considerada a que confere maior cardioproteção, independente do IMC e da idade (40). A um aumento de $1 \mathrm{mg} / \mathrm{dL}$ na concentração de HDL colesterol corresponderia uma redução de $3,2 \%$ no risco de doença coronariana, em mulheres (41).

Os efeitos da terapia hormonal sobre os lipídios circulantes variam de acordo com os produtos farmacêuticos empregados e sua via de administração. A primeira passagem pelo fígado, proporcionada pela ingestão oral de estrogênios, favorece a redução dos níveis de colesterol total, de LDL colesterol e da $\mathrm{Lp}(\mathrm{a})$ e o aumento dos níveis de HDL colesterol, mas também promove efeitos indesejáveis, como aumento da trigliceridemia e aumento dos fatores de coagulação (18).

A hipertrigliceridemia foi identificada em $56,9 \%$ de 325 mulheres equatorianas na pós-menopausa (42). Quando os níveis sanguíneos de triglicerídeos estão elevados, deve-se preferir a via não oral para a terapia hormonal (transdérmica).

Os progestógenos, utilizados obrigatoriamente nas mulheres não histerectomizadas, para proteção endometrial, também podem interferir nos efeitos benéficos da estrogenioterapia. Os progestógenos com ação androgênica, como o levonorgestrel (43), ou glicocorticoide, como o acetato de medroxiprogesterona (44), podem antagonizar os benefícios dos estrogênios, o que não se observa com a progesterona micronizada (45), com a didrogesterona (46) ou com a drospirenona (47).

A melhora dos fatores aterogênicos com a terapia hormonal se reflete em benefícios na prevenção de eventos cardiovasculares, avaliada por exames de imagem. A evolução da aterosclerose, mensurada pelo estudo da relação da espessura íntima/média carotídea, em mulheres na pós-menopausa saudáveis, foi mais lenta nas tratadas com $1 \mathrm{mg}$ de 17 -beta estradiol por via oral, do que em mulheres sem terapia hormonal (48). Um estudo randomizado e controlado com placebo em 1.064 mulheres na pós-menopausa sem alterações nas artérias coronárias, com idade entre 50 e 59 anos, mostrou que o uso de estrogênios evitou a calcificação co- ronariana (49). Nas portadoras de coronariopatia estabelecida, entretanto, não houve melhora da progressão da aterosclerose coronariana, quer com uso exclusivo de estrogênios, quer associados a progestógenos (50), podendo, inclusive, haver aumento de $29 \%$ no risco de infarto do miocárdio em mulheres sob terapia combinada de estrógeno e progestógeno, com média de idade de 63 anos, como demonstrou o estudo Women's Health Initiative (WHI) (51). Uma recente reanálise dos dados desse estudo mostrou que a presença de síndrome metabólica antes do início do tratamento aumenta os riscos de desfechos coronarianos desfavoráveis com a terapia hormonal (52).

\section{HIPERTENSÃO ARTERIAL}

É frequente o aparecimento de hipertensão arterial após a menopausa (53). Um estudo de 325 mulheres na pós-menopausa, realizado no Equador, identificou uma prevalência de $38,8 \%$ de hipertensão (42). A base fisiopatológica da hipertensão arterial após a menopausa ainda não está bem estabelecida, havendo diversas hipóteses para explicá-la: aumento da relação androgênio/estrogênio, alteração no sistema renina-angiotensina, aumento da endotelina, estresse oxidativo, obesidade e ativação do sistema nervoso simpático (54). Sugere-se, também, uma participação do peptídeo natriurético atrial (ANP), que diminui com a redução dos estrogênios produzidos pelos ovários, situação que pode ser revertida pela estrogenioterapia, como foi demonstrado em camundongos (55).

A experiência com anovulatórios para evitar a gravidez gerou a preocupação de que a terapia hormonal da menopausa também pudesse causar aumento da pressão arterial. Os contraceptivos orais podem promover aumento da pressão arterial sistólica e diastólica em algumas mulheres, independente do progestógeno em sua composição, mesmo na semana de interrupção do seu uso (56). Os hormônios utilizados nesses compostos, entretanto, são diferentes dos recomendados para a terapia hormonal da menopausa. $\mathrm{Na}$ imensa maioria deles, o estrógeno é o etinilestradiol, muito mais potente do que o 17-beta estradiol, e o progestógeno geralmente tem propriedades bem diferentes da progesterona produzida pelos ovários, podendo ter efeitos androgênicos, antiandrogênicos, glicocorticoides e antimineralocorticoides (57).

Uma revisão de 19 estudos sobre os efeitos da terapia hormonal da menopausa na pressão arterial de mu- 
lheres normo e hipertensas mostrou, em cinco deles, que a pressão arterial não se alterou, e em 14 houve diminuição dos níveis tensionais, sendo a redução mais observada com o uso de terapia transdérmica $(11 \mathrm{em}$ 13 estudos) do que com o emprego da via oral (quatro em 11 estudos) (58). Em um grupo de 1.397 mulheres na pós-menopausa, hipertensas, que usou terapia hormonal da menopausa por via transdérmica, foi observada uma redução média de $7 \mathrm{mmHg}$, na pressão arterial sistólica, e de $9 \mathrm{mmHg}$ na diastólica (59).

É mais indicado o uso de estrogênios por via não oral em mulheres hipertensas, pois dessa forma se evitam os efeitos da primeira passagem pelo fígado sobre o sistema renina-angiotensina, preservando-se os benefícios sobre a função endotelial (18). A terapia hormonal transdérmica diminui a atividade simpática, contribuindo, assim, para redução da pressão arterial (60).

\section{CONSIDERAÇÕES FINAIS}

Na mulher na pós-menopausa com síndrome metabólica, a primeira abordagem terapêutica deve ser a promoção de hábitos saudáveis de vida, com perda de peso e um programa regular de atividade física. Os diversos componentes da síndrome metabólica devem merecer atenção individual, com uso de medicamentos para hipertensão arterial, dislipidemia e diabetes, quando necessário. Quando os sintomas de síndrome do climatério estiverem presentes, a terapia hormonal, individualizada de acordo com a vontade da paciente e suas características clínicas, pode contribuir também para a melhora do quadro metabólico e do risco cardiovascular, desde que instituída nos primeiros anos após a menopausa.

Declaração: os autores declaram não haver conflitos de interesse científico neste estudo.

\section{REFERÊNCIAS}

1. StangIV, Baumann G, Stangl K. Coronary atherogenic risk factors in women. Eur Heart J. 2002;23:1738-52.

2. Kannel WB, Hjortland MC, McNamara PM, Gordon T. Menopause and risk of cardiovascular disease: the Framingham study. Ann Intern Med. 1976;85:447-52.

3. Lin JW, Caffrey JL, Chang MH, Lin YS. Sex, menopause, metabolic syndrome, and all-cause and cause-specific mortality-cohort analysis from the Third National Health and Nutrition Examination Survey. J Clin Endocrinol Metab. 2010;95:4258-67.

4. Deibert P, Konig D, Vitolins MZ, Landmann U, Frey I, Zahradnik $H P$, et al. Effect of a weight loss intervention on anthropometric measures and metabolic risk factors in pre- versus postmenopausal women. Nutr J. 2007;6:31.
5. Eshtiaghi R, Esteghamati A, Nakhjavani M. Menopause is an independent predictor of metabolic syndrome in Iranian women. Maturitas. 2010;65:262-6.

6. Heidari R, Sadeghi M, Talaei M, Rabiei K, Mohammadifard N, Sarrafzadegan N. Metabolic syndrome in menopausal transition: Isfahan Healthy Heart Program, a population based study. Diabetol Metab Syndr. 2010;2:59.

7. Kim HM, Park J, Ryu SY, Kim J. The effect of menopause on the metabolic syndrome among Korean women: the Korean National Health and Nutrition Examination Survey, 2001. Diabetes Care. 2007;30:701-6.

8. Pandey S, Srinivas M, Agashe S, Joshi J, Galvankar P, Prakasam $\mathrm{CP}$, et al. Menopause and metabolic syndrome: a study of 498 urban women from western India. J Midlife Health. 2010;1:63-9.

9. Carr MC. The emergence of the metabolic syndrome with menopause. J Clin Endocrinol Metab. 2003;88:2404-11.

10. Kahn R, Buse J, Ferrannini E, Stern M. The metabolic syndrome: time for a critical appraisal: joint statement from the American Diabetes Association and the European Association for the Study of Diabetes. Diabetes Care 2005;28:2289-304.

11. Wamala SP, Lynch J, Horsten M, Mittleman MA, SchenckGustafsson K, Orth-Gomer K. Education and the metabolic syndrome in women. Diabetes Care. 1999;22:1999-2003.

12. Pi-Sunyer $X$. The metabolic syndrome: how to approach differing definitions. Med Clin North Am. 2007;91:1025-40, vii.

13. Alberti KG, Zimmet PZ. Definition, diagnosis and classification of diabetes mellitus and its complications. Part 1: diagnosis and classification of diabetes mellitus provisional report of a WHO consultation. Diabet Med. 1998;15:539-53.

14. Gaspard U. Hyperinsulinaemia, a key factor of the metabolic syndrome in postmenopausal women. Maturitas. 2009;62:362-5.

15. Reaven GM. Banting lecture 1988. Role of insulin resistance in human disease. Diabetes. 1988;37:1595-607.

16. Reaven G. Metabolic syndrome: pathophysiology and implications for management of cardiovascular disease. Circulation. 2002;106:286-8.

17. Oliveira JE, Milech A, Franco LJ. The prevalence of diabetes in Rio de Janeiro, Brazil. The Cooperative Group for the Study of Diabetes Prevalence in Rio De Janeiro. Diabetes Care. 1996;19:663-6.

18. Stevenson JC. Type and route of estrogen administration. Climacteric. 2009;12 Suppl 1:86-90.

19. Expert Panel on Detection E, Treatment of High Blood Cholesterol in A. Executive Summary of The Third Report of The National Cholesterol Education Program (NCEP) Expert Panel on Detection, Evaluation, and Treatment of High Blood Cholesterol In Adults (Adult Treatment Panel III). JAMA. 2001;285:2486-97.

20. Alberti KGMM, Zimmet $P$, Shaw J. Metabolic syndrome-a new world-wide definition. A Consensus Statement from the International Diabetes Federation. Diabet Med. 2006;23:469-80.

21. Guzik TJ, Mangalat D, Korbut R. Adipocytokines - novel link between inflammation and vascular function? J Physiol Pharmacol. 2006;57:505-28.

22. Chu MC, Cosper P, Orio F, Carmina E, Lobo RA. Insulin resistance in postmenopausal women with metabolic syndrome and the measurements of adiponectin, leptin, resistin, and ghrelin. Am J Obstet Gynecol. 2006;194:100-4.

23. Kim JH, Kim SH, Im JA, Lee DC. The relationship between visfatin and metabolic syndrome in postmenopausal women. Maturitas. 2010;67:67-71.

24. Manolopoulos KN, Karpe F, Frayn KN. Gluteofemoral body fat as a determinant of metabolic health. Int $\mathrm{J}$ Obes. (Lond) 2010;34(6):949-59. 
25. Rebuffe-Scrive M, Eldh J, Hafstrom LO, Bjorntorp P. Metabolism of mammary, abdominal, and femoral adipocytes in women before and after menopause. Metabolism. 1986;35:792-7.

26. Cooke PS, Naaz A. Role of estrogens in adipocyte development and function. Exp Biol Med (Maywood). 2004;229:1127-35.

27. Ley CJ, Lees B, Stevenson JC. Sex- and menopause-associated changes in body-fat distribution. Am J Clin Nutr. 1992;55:950-4.

28. Crawford SL, Casey VA, Avis NE, McKinlay SM. A longitudinal study of weight and the menopause transition: results from the Massachusetts Women's Health Study. Menopause. 2000;7:96-104.

29. Haarbo J, Marslew U, Gotfredsen A, Christiansen C. Postmenopausal hormone replacement therapy prevents central distribution of body fat after menopause. Metabolism. 1991;40:1323-6.

30. Poehlman ET, Tchernof A. Traversing the menopause: changes in energy expenditure and body composition. Coron Artery Dis. 1998;9:799-803.

31. Bianchini F, Kaaks R, Vainio H. Overweight, obesity, and cancer risk. Lancet Oncol. 2002;3:565-74.

32. Kabat GC, Kim M, Chlebowski RT, Khandekar J, Ko MG, McTiernan $A$, et al. A longitudinal study of the metabolic syndrome and risk of postmenopausal breast cancer. Cancer Epidemiol Biomarkers Prev. 2009;18:2046-53.

33. Rosato V, Bosetti C, Talamini R, Levi F, Montella M, Giacosa $A$, et al. Metabolic syndrome and the risk of breast cancer in postmenopausal women. Ann Oncol. 2011;22:2687-92.

34. Albala C, Yanez M, Devoto E, Sostin C, Zeballos L, Santos JL. Obesity as a protective factor for postmenopausal osteoporosis. Int J Obes Relat Metab Disord. 1996;20:1027-32.

35. Jouyandeh Z, Nayebzadeh F, Qorbani M, Asadi M. Metabolic syndrome and menopause. J Diabetes Metab Disord. 2013;12:1.

36. Klein S. Outcome success in obesity. Obes Res. 2001;9 Suppl 4:354S-8S.

37. Ryan AS, Pratley RE, Goldberg AP, Elahi D. Resistive training increases insulin action in postmenopausal women. $\mathrm{J}$ Gerontol A Biol Sci Med Sci. 1996;51:M199-205.

38. Cornu C, Mercier C, French P, Bully C, Pugeat M, Cousin P, et al. Postmenopause hormone treatment in women with NIDDM or impaired glucose tolerance: the MEDIA randomized clinical trial. Maturitas. 2000;37:95-104.

39. Gaspard UJ, Gottal JM, Vandenbrule FA. Postmenopausal changes of lipid and glucose metabolism: a review of their main aspects. Maturitas. 1995;21:171-8.

40. Stevenson JC, Crook D, Godsland IF. Influence of age and menopause on serum lipids and lipoproteins in healthy women. Atherosclerosis. 1993;98:83-90.

41. Gordon DJ, Probstfield JL, Garrison RJ, Neaton JD, Castelli WP, Knoke JD, et al. High-density lipoprotein cholesterol and cardiovascular disease. Four prospective American studies. Circulation. 1989;79:8-15.

42. Hidalgo LA, Chedraui PA, Morocho N, Alvarado M, Chavez D, Huc A. The metabolic syndrome among postmenopausal women in Ecuador. Gynecol Endocrinol. 2006;22:447-54.

43. Hirvonen E, Lipasti A, Malkonen M, Karkkainen J, Nuntila J, Timonen $\mathrm{H}$, et al. Clinical and lipid metabolic effects of unopposed oestrogen and two oestrogen-progestogen regimens in postmenopausal women. Maturitas 1987;9:69-79.
44. Aygen EM, Basbug M, Tayyar M, Kaya E. The effects of different doses of medroxyprogesterone acetate on serum lipids, lipoprotein levels and atherogenic index in the menopausal period. Gynecol Endocrinol 1998;12:267-72.

45. Moorjani S, Dupont A, Labrie F, De Lignieres B, Cusan L, Dupont $P$, et al. Changes in plasma lipoprotein and apolipoprotein composition in relation to oral versus percutaneous administration of estrogen alone or in cyclic association with utrogestan in menopausal women. J Clin Endocrinol Metab. 1991;73:373-9.

46. Kuba VM, Teixeira MA, Meirelles RM, Assumpcao CR, Costa OS. Dydrogesterone does not reverse the cardiovascular benefits of percutaneous estradiol. Climacteric. 2012;16:54-61.

47. Shulman LP. A review of drospirenone for safety and tolerability and effects on endometrial safety and lipid parameters contrasted with medroxyprogesterone acetate, levonorgestrel, and micronized progesterone. J Womens Health (Larchmt). 2006;15:584-90.

48. Hodis HN, Mack WJ, Lobo RA, Shoupe D, Sevanian A, Mahrer $\mathrm{PR}$, et al. Estrogen in the prevention of atherosclerosis. A randomized, double- blind, placebo-controlled trial. Ann Intern Med. 2001;135:939-53.

49. Manson JE, Allison MA, Rossouw JE, Carr JJ, Langer RD, Hsia J, et al. Estrogen therapy and coronary-artery calcification. $\mathrm{N}$ Engl $\mathrm{J}$ Med. 2007;356:2591-602.

50. Herrington DM, Reboussin DM, Brosnihan KB, Sharp PC, Shumaker SA, SnyderTE, et al. Effects of estrogen replacement on the progression of coronary-artery atherosclerosis. N Engl J Med. 2000;343:522-9.

51. Writing Group for the Women's Health Initiative Investigators. Risks and Benefits of Estrogen Plus Progestin in Healthy Postmenopausal Women. JAMA. 2002;288:321-33.

52. Wild RA, Wu C, Curb JD, Martin LW, Phillips L, Stefanick M, et al. Coronary heart disease events in the Women's Health Initiative hormone trials: effect modification by metabolic syndrome: a nested case-control study within the Women's Health Initiative randomized clinical trials. Menopause. 2013;20:254-60.

53. Beaufils M. [Hypertension in women after menopause]. Presse Med. 2006;35:1055-60.

54. Reckelhoff JF, Fortepiani LA. Novel mechanisms responsible for postmenopausal hypertension. Hypertension. 2004;43:918-23.

55. Belo NO, Sairam MR, Dos Reis AM. Impairment of the natriuretic peptide system in follitropin receptor knockout mice and reversal by estradiol: implications for obesity-associated hypertension in menopause. Endocrinology. 2008;149:1399-406.

56. Nichols M, Robinson G, Bounds W, Newman B, Guillebaud J. Effect of four combined oral contraceptives on blood pressure in the pill-free interval. Contraception. 1993;47:367-76.

57. Wiegratz I, Kuhl H. Progestogen therapies: differences in clinical effects? Trends Endocrinol Metab. 2004;15:277-85.

58. Mueck AO, Seeger H. Effect of hormone therapy on BP in normotensive and hypertensive postmenopausal women. Maturitas. 2004;49:189-203.

59. Mueck AO. Hypertension and HRT. Int Congr Ser. 2012;1229:115-22.

60. Vongpatanasin W, Tuncel M, Mansour Y, Arbique D, Victor RG. Transdermal estrogen replacement therapy decreases sympathetic activity in postmenopausal women. Circulation. 2001;103:2903-8. 